\title{
Implementation of IoT Frameworks in Business Process Automation
}

\author{
Shiv Kumar Jha ${ }^{1}$, Md. Omair Ahmad ${ }^{2}$, Shakeel Ahmad Siddiqui ${ }^{3}$, Jawed Ahmed ${ }^{5}$, Parul \\ Agarwal $^{5}$ \\ \{ shivkjha29@gmail.com ${ }^{1}$, omair4ahmad@gmail.com², \\ siddiquishakeelahmad@gmail.com ${ }^{3}$, jawed2047@gmail.com ${ }^{4}$, \\ pagarwal@jamiahamdard.ac.in\}
}

Jamia Hamdard

\begin{abstract}
Internet of Things brings different computing devices on a singular platform enabling data transfer, monitoring and analysis without human intervention. It is the next big frontier in expediting business processes. Business Process Automation (BPA) is a process of managing information, data and processes to reduce costs, resources and investment to achieve targeted results consistently aligned to the goals of an organisation. BPA makes processes more efficient by automating important tasks using technology. The implementation of IoT varies from industry to industry. BPA can leverage the true potential of IoT by bringing together data, machinery and customer experience.
\end{abstract}

Keywords: Business Process Automation, Business Process Management, sensors, Internet of Things.

\section{Introduction}

Nowadays, Internet of Things (IoT) has been disrupting the traditional work methodologies around the world. With plenty of experiments and research going on around it, in the near future it is expected to play a pivotal role in various different sectors which may include the manufacturing sector, logistics sector, clean energy, safety and security, logistics. With the fusion of Internet of Things in these areas it will have a huge impact on the livelihood of the general masses and they will change for the better. Along with the lifestyle changes of the public, new business models are also set to emerge [1]. In order to make this a reality we will need to set up robust frameworks that are highly secure and can be relied upon which will serve as a global platform for inter organisatonal collaboration. A distinct set of concepts, methodologies and standards need to be brought in place in order to device such infrastructures which are minful of the IoT and facilitiate its configuration and monitoring in a smooth yet highly effective manner. With the exponential rise in the number of devices in this decade (which is around fivefold, close to 75 billion!) there is a pressing need Canny frameworks with generous self-setup, self-observing, and self-recuperating properties are required to deal with the huge and quickly developing number of gadgets. Advancement is being made in a significant number of these territories; this position paper will along these lines concentrate on an issue that has not gotten enough consideration yet, however is urgent for structure and conveying IoT applications in modern or endeavour settings on a more extensive scale: The possible usage IoT in business operations mindful of IoT. 


\section{Administration Enablement of IoT}

As of now, most of the enterprise systems are not designed in a manner to interact with IoT devices like RFID and others, they heavily rely on the software side of computing and human intervention is required time and again. In order to leverage the inherent capabilities of IoT, it is essential to re-design if not replace, the pre-existing frameworks to institutionalise the Internet of Things by making them compatible with devices like sensors, actuators, RFID and other embedded devices etc. In order to enable smooth data transfer and communication across the network, Web Services [2] can be used. This means that there will be a web-based interface like a portal or an application that will allow human - machine intercation which also provides a user interface for end-users. Another way of implementing this could be by using Representational State Transfer (REST) based approach [3] which also comes under Web Services but allows the formation of more scalable frameworks because of its layered system, cacheabiilty and statelessnes.

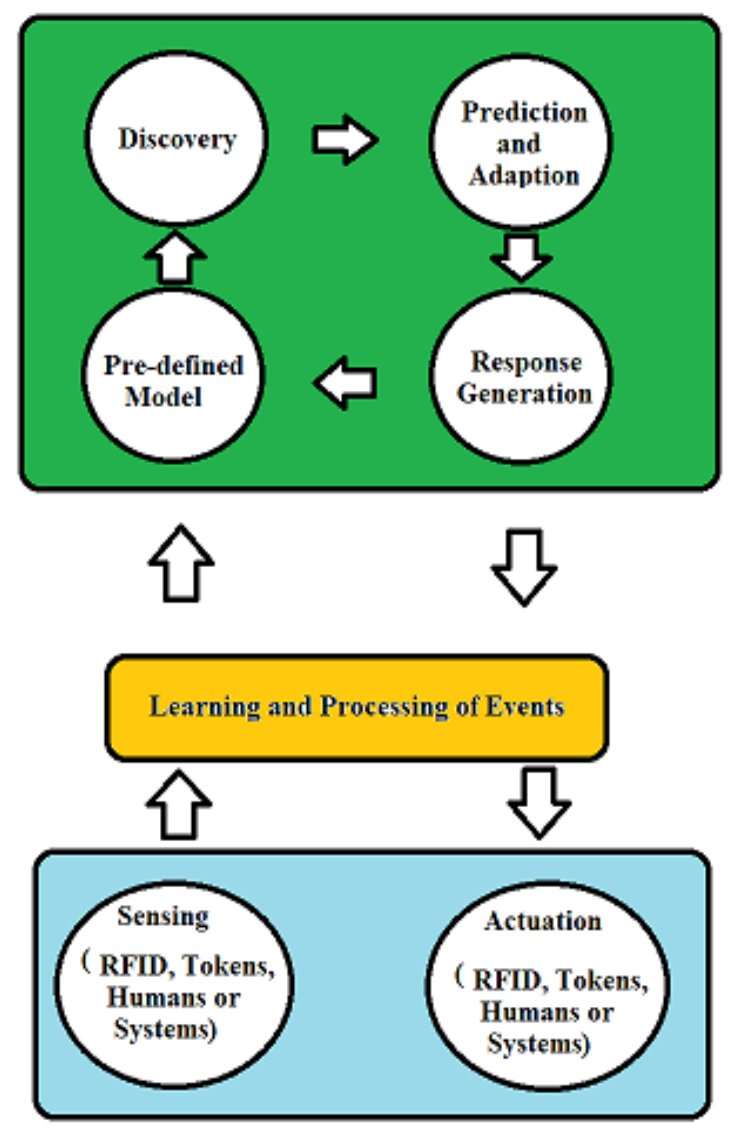

Fig. 1 An Overview of the Interaction Between BPM and IoT 
In order to minimise human intervention we need to adopt a different approach in IoT based business process models than the ones we are currently witnessing. This will lead to a drastic change in the implementation of BPM on technical grounds as well as the grounds of interconnectivity and fuctioning in general. With fast paced changes that we get to see, this infrastructure should also be well prepared to take into account any anomaly that may arise and take care of it by eaither resolving it or puting it into quarantine. Proper management of data is also an issue of great concern, but before we delve into it, it is essential to know and authenticate the origin of data beforehand that is parsed in order to avoid any risk on grounds of cyber security that may put the enterprise in danger; which may include but are not limited to insecure desrialisation, buffer overflow etc. Also, the place of service execution should be taken care of, REST-based methodologies are of great help in such cases where a proxy or any kind of a load balancer helps in masking the communicattion medium so that the end user does not get to know if it is in direct contact with the server or not. Last but not the least we also need to take care of trivial factors of error generation like poor caliberation of the sensors[4] and other devices, power exhaustion, signal strength and signal range, server issues etc. It is essential because while achieving operational efficiency, IoT also plays a role in fabricating new business models[5] like monetising user data and many others at the same time it also creates new and enhanced customer experiences as shown in Figure 2[6].

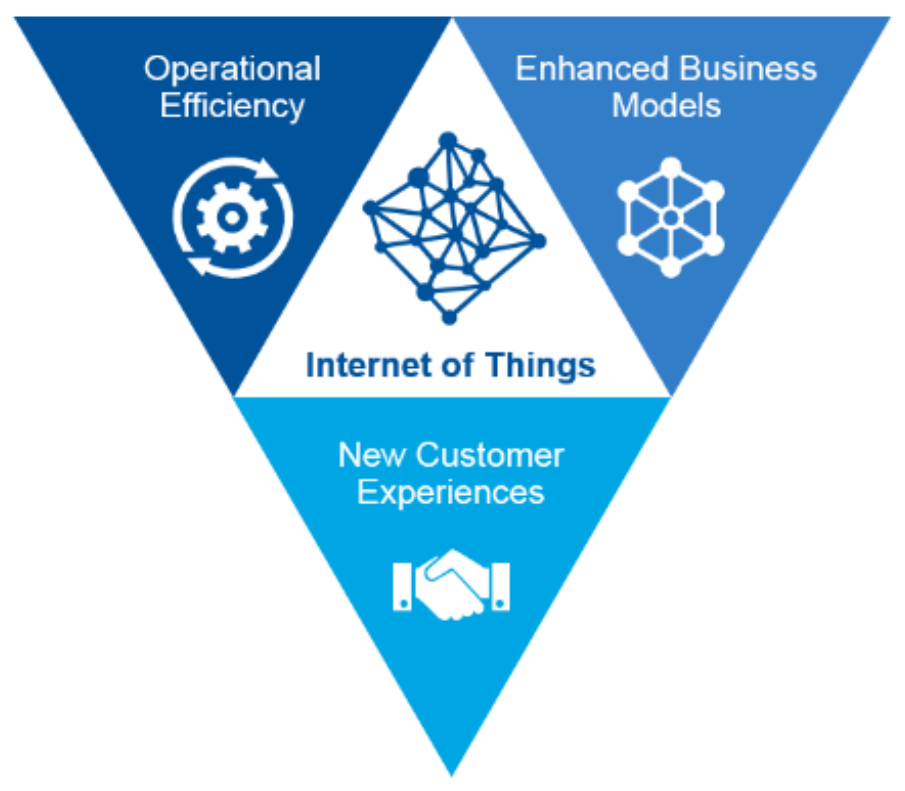

Fig. 2 Business Impact of IoT 


\section{Demonstration of IoT Mindful Business Processes}

Although business Process Management (BPM) [7] sytems are responsible for efficent demonstration and execution of complex tasks, the same does not hold true when it comes to IoT Mindful Business Processes. This may be one of the downside of such an infrastructure, setting up such a system in itself requires plenty of capital in hand, upront payments and various risks too in terms of functionality and maintenance apart from the initial struggles of engineering factors and customisation when dealing with sensors, RFID tags, actuators etc. Once deployed appropriately, they are forcasted to outrun and outperform the traditional BPM in the enterprises. Figure 3 shows a pedagogy of implementation of IoT architechure in business process automation.

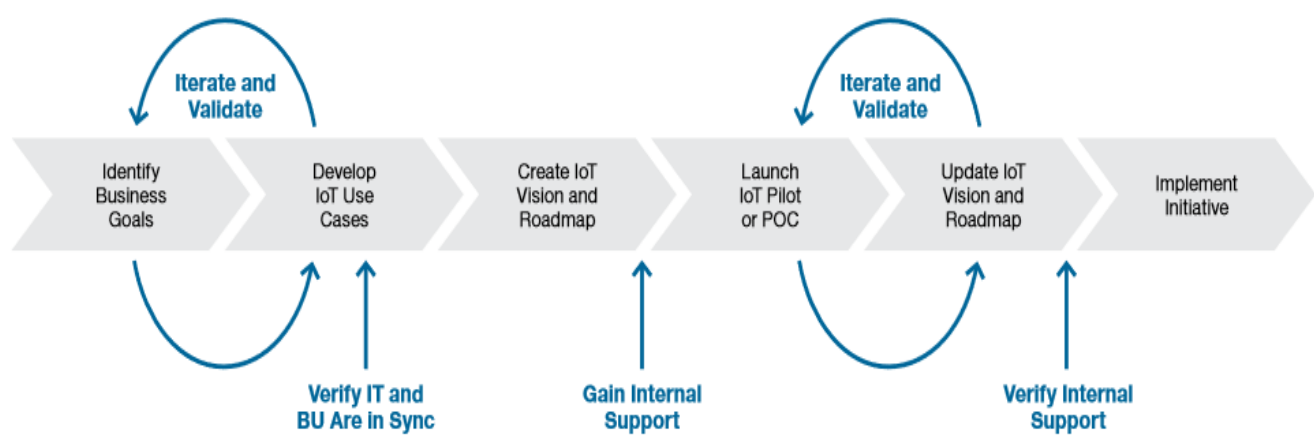

Fig 3. Implementation of IoT in Business Process Automation

These days, business processes seem to be headed towards a very planned approach and risk averse by bringing down the randomness involved in such processes to a nominal extent. Although there are various mechanisms in place to tackle this, but none seem to address the challenges proposed by IoT -mindful systems. Given below are some dominant issues that we need to address:

\subsection{Versatile and occasion driven procedures}

One of the fundamental advantages of using IoT is that procedures moved toward becoming more and more oriented, pro-active and hence responsive to the real world scenario. Although, this is heavily dependent on the response and real time analysis of the data that is generated by the sensors or by directly detecting such things for which it must be prepared in advance keeping in mind that some of these events are least probable to happen but are necessary to be detected if they do. For a portion of the occasions, the event likelihood is low. However, one realizes that they may happen, in an event that by any stretch of someone's imagination.

Demonstrating such occasions into a procedure is lumbering, as they would need to be incorporated into all conceivable exercises, prompting extra multifaceted nature and making it progressively hard to comprehend the demonstrated procedure, in specific the primary 
progression of the procedure (the $80 \%$ case). Besides, how to respond on a solitary occasion can rely upon the unique circumstance, for example the arrangement of occasions that have been distinguished already. A basic model: If individuals had entered a certain territory, and later a sharp ascent in temperature is distinguish there, just as smoke, at that point the salvage group needs to be sent. Or then again, if the truck is postponed with the goal that the conveyance can't arrive at the proposed B client in time, and if the organization simply has gotten a dire request from its favoured A client, at that point the truck is rerouted to the $\mathrm{A}$ client. [8]

\subsection{Procedures managing questionable information}

When we are taking into account the data that orginates from sensors which may give out a high discrepancy rate because of various physical factors, we need to have a certain check for its quality, i.e, a mode of authenticating how reliable or how vulnerable the data is. In this case we associate such data with Quality of Information (QoI) which ranges from anywhere between $0 \%$ to $100 \%$ on a comparative scale. In straightforward cases, this permits the procedure modeler to characterize limits: e.g., on the off chance that the level of sureness is over $90 \%$, at that point it is expected that the occasion truly occurred. In the event that it is somewhere in the range of half and $90 \%$, some different exercises will be activated to decide whether the occasion happened or not. In the event that it is underneath half, the occasion is disregarded. Things get increasingly mind boggling when numerous occasions are included: E.g., one occasion with $93 \%$ assurance, one with $75 \%$, and another with $49 \%$. The fundamental administrations that fire the first occasions must be modified to join such QoI qualities to the occasions. From a BPM point of view, it is however necessitated that such information can be caught, handled and communicated in the utilized demonstrating documentation language, for example BPMN. Furthermore, the grammar and semantics of such QoI qualities should be institutionalized: Is it a basic assurance rate as in the models above, or should it be something progressively expressive[9] (e.g., a run inside which the genuine worth falsehoods)?

\subsection{Procedures managing inconsistent assets}

Not just the data from various assets is naturally vulnerable and hence cannot be relied upon blindly, yet additionally the assets giving the data themselves, e.g., because of the improper functioning of the facilitating gadget. Procedures depending on such assets should most likely adjust to such circumstances. The main issue is to distinguish such a disappointment by any stretch of the imagination: In the case that a procedure is calling an asset straightforwardly[10], this location is inconsequential. When we're discussing assets that might produce an occasion at one point in time (e.g., the asset that screens the temperature condition inside the truck and sends a caution on the off chance that it has turned out to be excessively hot), it is increasingly troublesome: Not having gotten any occasion can be on account of asset disappointment, yet additionally in light of the fact that there was nothing to report. Some observing programming is expected to identify such issues; it is misty however in the event that such programming ought to be a piece of the BPM execution condition or ought to be a different part.

\subsection{Exceptionally diverse procedures}


When cooperation with true items and gadgets is required, it can make sense to execute a procedure in a decentralized manner. As expressed in [1], the disintegration and decentralization of existing business procedures builds adaptability and execution, could lead to various new streams of generating manifold revenue than the present ones by inculcating better mechanism in place for decision making through privilege the board of programming items sent on brilliant things. For instance, in natural observing or store network following applications, no messages should be sent to the focal framework as long as everything is inside as far as possible. Just if there is a deviation, and alarm (occasion) should be created, which thusly can prompt an adjustment of the general procedure. From a business procedure demonstration point of view however, it should be conceivable to characterize the procedure midway, including the way that a few exercises (i.e., the checking) will be done remotely. When the total procedure is demonstrated, it should then be conceivable to convey the related administrations to where they must be executed, and afterward run and screen the total process. [11]

\section{Conclusion}

In order to achieve good results in terms of effciency, reliability and pro-activeness we need to stablish a full blown IoT based infrastructure by bringing Business Process Modelling and IoT on the same page using Web Services or REST- based approach. If we are unable to do so, IoT will be limited to a mere vertical of engineering although it has the capacity to usher growth in various different arenas. While doing so, it is also important to keep in mind the various risk factors involved along with hadling anomalies in the process which can only be dealt with proper testing snd experimentation; it would not be wrong to say that IoT still has a long way to go. When doing as such, the unique attributes of IoT administrations and procedures must be considered and likely existing business procedure displaying and execution dialects like BPMN [12] and WS-BPEL [13], just as administration depiction dialects like USDL [14], should be broadened. With respect to displaying of dispersed procedures, in an initial step it should be conceivable to characterize in the demonstrating condition which exercises should be tested in which kind of environments which proper testing methodologies in place, and to choose the administrations that actualize these remote exercises. When it comes to the implementation stage in conditions where the system has various constaraints on connectivity due to devices and environment, REST always seems to have an edge over other web services like SOAP. Another way of implementing is by using the models to deploy services too, this looks like a more advanced approach than the one stated earlier. It invloves sending an entire sub-process to a remote BPM which then implements it on its own, this can happen only if all the services and/or packages that are required are already sent to tha target devices where it is to be executed. At a later stage we may go further to either semi-sutomate or totally automate the decision making process so that the system is capable of deciding which process needs to be deployed where.

\section{References}


[1] O. Vermesan, M. Harrison, H. Vogt, K. Kalaboukas, M. Tomasella et al. (Eds.), "The Internet of Things - Strategic Research Roadmap", Cluster of European Research Projects on the Internet of Things, CERP-IoT, 2009.

[2] W3C, Unified Service Description Language Incubator Group, http://www.w3.org/2005/Incubator/usdl/

[3] D. Guinard, V. Trifa, E. Wilde, "A Resource Oriented Architecture for the Web of Things", in Proceedings of Internet of Things 2010 International Conference (IoT 2010). Tokyo, Japan, November 2010.

[4] You, K., Yang, W., \& Han, R. (2015). The video collaborative localization of a miner's lamp based on wireless multimedia sensor networks for underground coal mines. Sensors, 15(10), 25103-25122.

[5] Liu, C., Li, Q., \&amp; Zhao, X. (2009). Challenges and opportunities in collaborative business process management: Overview of recent advances and introduction to the special issue. Information Systems Frontiers, 11(3), 201-209.

[6] Gartner Trend insight Report, Published: 22 September, 2017; ID: G00333540

https://emtemp.gcom.cloud/ngw/globalassets/en/doc/documents/3806366-business-

benefits-of-the-internet-of-things-a-gartner-trend-insight-report.pdf

[7] Cheng, M. Y., Chiu, K. C., Hsieh, Y. M., Yang, I. T., Chou, J. S., \& Wu, Y. W. (2017).

BIM integrated smart monitoring technique for building fire prevention and disaster relief. Automation in Construction, 84, 14-30.

[8] Smirnov, S., Reijers, H. A., Weske, M., \& Nugteren, T. (2012). Business process model abstraction: a definition, catalog, and survey. Distributed and Parallel Databases, 30(1), 6399.

[9] Meyer, S., Ruppen, A., \& Magerkurth, C. (2013, June). Internet of things-aware process modelling: integrating IoT devices as business process resources. In International conference on advanced information systems engineering (pp. 84-98). Springer, Berlin, Heidelberg.

[10] Schulte, S., Janiesch, C., Venugopal, S., Weber, I., \& Hoenisch, P. (2015). Elastic Business Process Management: State of the art and open challenges for BPM in the cloud. Future Generation Computer Systems, 46, 36-50 .

[11] Cheng, Y., Zhao, S., Cheng, B., Chen, X., \& Chen, J. (2019). Modeling and Deploying IoT-Aware Business Process Applications in Sensor Networks. Sensors, 19(1), 111.

[12] OMG, Business Process Model and Notation specification, available at http://www.omg.org/technology/documents/br_pm_spec_catalog.htm

[13] OASIS, Web Services Business Process Execution Language, http://docs.oasisopen.org/wsbpel/2.0/wsbpel-v2.0.html

[14] W3C, Unified Service Description Language Incubator Group, http://www.w3.org/2005/Incubator/usdl/ 